\title{
Phases of Bosons or Fermions in confined optical lattices
}

\author{
H. Heiselberg \\ Univ. of S. Denmark, Campusvej 55, DK-5230 Odense M, Denmark
}

\begin{abstract}
Phases of Bose or Fermi atoms in optical lattices confined in harmonic traps are studied within the Thomas-Fermi approximation. Critical radii and particle number for onset of Mott insulator states are calculated and phase diagrams shown in 1D, and estimated for 2 and 3D. Methods to observe these and novel phases such as d-wave superconductivity are discussed. Specifically the collective modes are calculated.

PACS numbers: 03.75.Ss, 71.10.Pm, 03.75.Lm
\end{abstract}

\section{INTRODUCTION}

Cold quantum systems in optical lattice have been realized with Bose and Fermi atoms, and superfluid transitions to Mott insulator (MI) solids at filling up to five [1-6]. Luttinger and Luther-Emery liquids and MI solids appear for 1D fermions whereas in 2D it is believed that d-wave superconductivity (dSC) appears between metal and antiferromagnetic (AFM) phases $[7,8]$, which is responsible for the high temperature superconductivity. Likewise quantum spin phases [9], supersolids [10], fractional quantum Hall phases $[11,12]$, mixed $[13]$ and other phases may be studied.

By tuning the barriers of the optical lattices and the interaction strengths near or far from Feshbach resonances, the Fermi [14] and Bose [15] Hubbard models can be realized in 1,2 and 3 dimensions $[16,17]$ for any interaction and hopping strengths. These are the on-site interaction energy $U$ and the hopping parameter $t$ to nearest site. Both can be expressed in terms of the lattice potential and particle interactions such as the s-wave scattering length (see, e.g. $[16,17])$. The optical lattices are in experiments usually confined in a trap typically in the form of a harmonic potential $V_{i}=V_{2} r_{i}^{2}$. Here, the lattice point $r_{i}=i d$ is an integer times the lattice spacing $d$. The optical lattices are then clean systems with controlled disorder. A number of MI, superfluid and other phases appear as the densities increase towards the center of the trap [16-22].

It is the purpose here to calculate the phase diagram for these models in the Thomas-Fermi approximation (TF) at zero temperature, which can be done analytically in $1 \mathrm{D}$ for strong and weak couplings $U / t$ and approximated between these limits. The corresponding phases in 2D and 3D will also be discussed. Finally, the 1D collective modes are calculated for bosons and fermions in harmonically confined optical lattices.

\section{EQUATION OF STATES IN THE THOMAS-FERMI LIMIT}

In the limit of many particles in a shallow confining harmonic potential, the TF (or local density approximation) applies because the length scales over which the trapping potential and density varies are long compared to phase boundaries [23] and the lattice spacing. As will be shown below, the size of the system scales as $R \sim \sqrt{t / V_{2}}$, and the total number of particles scales as $N \sim R / d$. Therefore the average filling fraction scales as $\sim N / R \simeq N d \sqrt{V_{2} / t}$, and can be held fixed in the TF limit: $N \rightarrow \infty$ and $V_{2} \rightarrow 0$.

In $\mathrm{TF}$ the total chemical potential is given by the sum of the trap potential and the local chemical potential $\mu(\rho)=d \varepsilon / d \rho$, where $\varepsilon$ and $\rho(r)$ are the local energy and density per site, i.e.

$$
\mu_{T o t}=\mu(\rho, U)+V_{2} r^{2}=\mu(0, U)+V_{2} R^{2} .
$$

It must be constant over the lattice and can therefore be set to its value at the edge or radius $R$ of the atomic gas, which gives the last equation in (1). As we shall see later $\mu(\rho=0, U)=-z t$ for both repulsive fermions and bosons, where $z=2 D$ is the number of neighbors in $D$ dimensions.

\section{A. 1D Fermions}

We start with a $1 \mathrm{D}$ system of spin-balanced fermions with repulsive interactions described by the Hubbard model [14]

$$
H=-t \sum_{\langle i j\rangle \sigma} \hat{a}_{i \sigma}^{\dagger} \hat{a}_{j \sigma}+U \sum_{i} \hat{n}_{i \uparrow} \hat{n}_{i \downarrow},
$$

where, $\hat{a}_{i \sigma}$ is the usual Fermi creation operators, $n_{i \sigma}=$ $\hat{a}_{i \sigma}^{\dagger} \hat{a}_{i \sigma}$ the density, and $\langle i j\rangle$ sums over nearest neighbors $j=i \pm 1$.

The ground state energy per site at zero temperature can be calculated [24] from the Bethe ansatz. A good approximation for densities $\rho \leq 1$ and all $U \geq 0$ is [19]

$$
\mu(\rho, U)=-2 t \cos (\pi \rho / \beta) .
$$

It is exact in both limits: $\beta(U \rightarrow \infty)=1$ and $\beta(U \rightarrow$ $0)=2$. For densities $1<\rho \leq 2$ the chemical potential is: $\mu(\rho, U)=U-\mu(2-\rho, U)$. [24] The phase is a band insulator (BI) for $\rho=2$, a MI for $\rho=1$ and a Luttinger liquid (LL) [25] otherwise. The MI phase has a chemical potential gap $\Delta \mu=U+4 t \cos (\pi / \beta)$. It must match [24] 


$$
\Delta \mu(U)=U-4 t+8 t \int_{0}^{\infty} \frac{J_{1}(x)}{x[1+\exp (x U / 2 t)]} d x,
$$

where $J_{1}$ is the 1 st Bessel function. Alternatively, one could as in [19] require that the energy: $\epsilon(1, U)=$ $-2 t(\beta / \pi) \sin (\pi / \beta)$, matches the exact result of the Bethe ansatz [24]. We prefer matching the chemical potentials because it insures that the Mott transitions are correct. The chemical potential is discontinuous at $\rho=1$ by an amount

$$
\Delta \mu=U-4 t+8 \ln (2) t^{2} / U+\mathcal{O}\left(U^{-2}\right),
$$

for $U \gg t$ whereas in the weakly repulsive limit the gap vanishes exponentially as

$$
\Delta \mu=\frac{-\sqrt{U t}}{\pi} \exp (-2 \pi t / U)
$$

The density is now found by inserting (3) in (1). For $\rho \leq 1$ we obtain

$$
\rho(r)=\frac{2 \beta}{\pi} \arcsin \left(\sqrt{R^{2}-r^{2}} / R_{c}\right),
$$

where $R_{c}=2 \sqrt{t / V_{2}}$. The gap in the chemical potential Eq. (1) requires a density plateau at $\rho=1$ [19-22]. We can now relate $\rho(0)$ to the radius $R$ and the corresponding total number of atoms

$$
N=\frac{2}{d} \int_{0}^{R} \rho(r) d r
$$

in the confined lattice for any $U / t$.

We are particularly interested in the critical radii $R_{n}^{ \pm}$ and the corresponding critical number of atoms $N_{n}^{ \pm}=$ $(2 / d) \int_{0}^{R_{n}^{ \pm}} \rho(r) d r$, right when the MI plateaus of filling $n$ first appear (-) in the center of the trap and disappear $(+)$ forming a MI shell. The MI phase with $\rho(0)=n=1$ first appear in the center of the trap when $N=N_{1}^{-}$and persist up to $N=N_{1}^{+}$where after the core is a LL with $\rho>1$ until $N=N_{2}^{-}$where a BI with $\rho=2$ forms in the core.

For $U \gg t(\beta \rightarrow 1)$ the MI first appear in the center when $R_{1}^{-}=R_{c}$ according to Eq. (7), and the corresponding number of particles is $N_{1}^{-}=N_{c}$, where

$$
N_{c}=\frac{4 R_{c}}{\pi d}=\frac{8}{\pi d} \sqrt{\frac{t}{V_{2}}} .
$$

Likewise, it follows that for $U \ll t$, where $\beta=2$ and $\Delta \mu=0$, the critical MI radius is shorter $R_{1}^{+}=R_{c} / \sqrt{2}$ whereas the $\mathrm{BI}$ radius is $R_{2}=R_{c}$. The corresponding critical number of atoms are

$N_{1}^{-}=N_{1}^{+}=\left(4 R_{c} / \pi d\right) \int_{0}^{1} \arccos \left(x^{2}\right) d x \simeq 0.847 N_{c}$,

and $N_{2}^{-}=2 N_{c}$.

Returning to $U \gg t$ we must add the chemical potential gap $\Delta \mu=U-4 t$ to Eq. (1) when $\rho(r)>1$. The critical radius, where then density just starts to exceed unity $\rho=1_{+}$in the core is: $R_{1}^{+}=R_{c} \sqrt{U / 4 t}$, whereas the BI with $\rho=2$ appears when $R_{2}^{-}=R_{c} \sqrt{U / 4 t+1}$. The corresponding number of atoms is

$$
N_{1}^{+}=\frac{2}{d} \sqrt{U / V_{2}}=N_{c} \frac{\pi}{4} \sqrt{U / t},
$$

and

$$
N_{2}^{-}=N_{c}\left[\frac{\pi}{4} \sqrt{U / t}+1\right] .
$$

We emphasize that the calculated values for the critical fillings $N_{1}^{-}, N_{1}^{+}$and $N_{2}^{-}$and the corresponding radii are exact in both the weak and strong coupling limits within TF.

For general $U$ we calculate $\Delta \mu(U)$ from Eq. (4) to find $\beta(U)$. Inserting (3) in (1) then gives the density, critical radii and the critical fillings as shown in Fig. 1. They interpolate smoothly between the analytical results $U \rightarrow 0$ and $U \gg t$ given above.

The numerical calculations in Refs. $[27,20]$ find similar phase diagrams as the TF continuum limit $N \rightarrow \infty$ discussed above, however, with a few important differences. The finite number of particles makes it impossible to resolve the exponentially small gap for $U \rightarrow 0$. The MI plateau in the density at $\rho=1$ does not extend over a sufficient number of lattice sites, $R_{1}^{+}-R_{1}^{-} \lesssim d$, and the phase boundary cannot be resolved. Therefore, the critical point where the $\rho=1 \mathrm{MI}$ state vanishes moves from $U_{1}=0$ in TF to $U_{1} \simeq 0.8 t$ in [20] and $U_{1} \simeq 3 t$ in [27]. As a consequence the phase diagrams of Refs. $[27,20]$ acquire an additional phase transition at $U_{1}$ and $N \gtrsim N_{1}^{-}$between phases with and without a "resolvable" $\rho=1$ Mott transition. A similar phenomenon will occur at finite temperature, where the MI solid melts and the critical point moves to a finite $U$ since $\Delta \mu(U) \simeq k_{B} T$.

The attractive Hubbard model $(U \leq 0)$ tends to pair atoms in a Luther-Emery liquid (LE) [26]. The trap potential breaks translational invariance forming an atomic density wave (ADW) phase with oscillating density: $\rho\left(r_{i}\right)=\bar{\rho}+\delta \rho \cos \left(2 \pi r_{i} / \lambda_{A D W}\right)[26,19,21]$. Here, $\bar{\rho}$ is the average density, $\delta \rho$ is the amplitude and $\lambda_{A D W}=$ $2 d / \bar{\rho}$ the wavelength. The TF can be applied for the average density $\bar{\rho}$ as long as $R \gg \lambda_{A D W}$. As described in [19] the parametrization

$$
\mu=U / 2-2 \alpha^{2} t \cos (\pi \bar{\rho} / 2),
$$

is a good approximation for the EoS at all densities and $U \leq 0$. It satisfies the known properties for weak and strong attraction [24], when the prefactor is chosen as: $\alpha^{2}(U)=\pi \int_{0}^{\infty} J_{0}(x) J_{1}(x) /(1+\exp (x|U| / 2))(d x / x)$. It decreases from $\alpha(U=0)=1$ to $\alpha(U \rightarrow-\infty)=$ $\sqrt{\pi \ln (2)} t /|U|$. No MI are formed in the LE at $\bar{\rho}=1$ whereas a BI appears when $\rho=2$ with radius $R_{2}=$ $2 \alpha t / V_{2}=\alpha R_{c}$. The corresponding number of particles is

$$
N_{2}^{-}=2 \alpha N_{c},
$$

which is also shown in Fig. 1. 


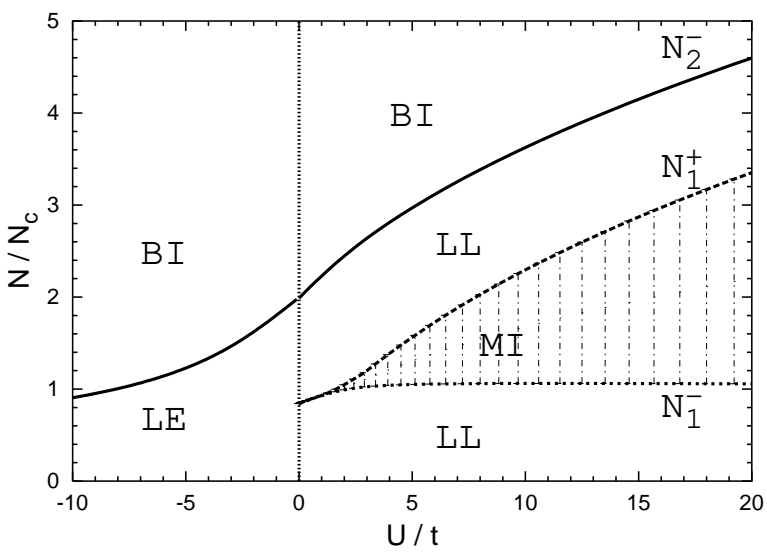

FIG. 1. Phase diagram of Fermions in a confined 1D optical lattice vs. $U / t$ and number of atoms. The phases are denoted by the core state. For example, for $U>0$ the BI phase has a band insulator core with $\rho=2$ ) surrounded by a LL layer with $1<\rho<2$ again surrounded by a MI layer with $\rho=1$ and finally a surface LL layer with $0 \leq \rho<1$. The curves separate the phases where the MI appear $\left(N_{1}\right)$, vanish $\left(N_{1}^{+}\right)$ and a BI $\left(N_{2}^{-}\right)$appear in the center (see text). For $U \leq 0$ the LE liquid has a BI core when $N>N_{2}^{-}$.

\section{B. 1D Bosons}

Bosons do not experience Pauli blocking and therefore have a rather different phase diagram as fermions even for the same lattice, particle mass and interactions. The Bose-Hubbard [15] is given by:

$$
H=-t \sum_{\langle i j\rangle} \hat{b}_{i}^{\dagger} \hat{b}_{j}+\frac{1}{2} U \sum_{i} \hat{n}_{i}\left(\hat{n}_{i}-1\right)
$$

where $\hat{b}_{i}$ is the Bose creation operator and $\hat{n}_{i}=\hat{b}_{i}^{\dagger} \hat{b}_{i}$ the density.

Superfluid transitions to MI solid phases appear at integer filling, $n=1,2,3, \ldots$, above a critical coupling $[27,28] U_{1}=3.84 t, U_{2}=6.2 t$, and $U_{n}=2.2 n t$ for $n \gg 1$. Just above a critical coupling a Mott gap opens in the chemical potential compatible with a Kosterlitz-Thouless transition: $\Delta \mu_{n} \sim \exp \left(-\right.$ const $\left./ \sqrt{U-U_{n}}\right)$. When $U \gg$ $U_{n}$ we have $\Delta \mu_{n}=U-2 z t$, whereas for $U \leq U_{n}$ there is no gap and $\Delta \mu_{n}=0$. A good approximation to numerical results [27] for $U \gg t$ is the simple parametrization

$$
\mu=n U+\left(U-\Delta \mu_{n}\right) \sin ^{2}[(\rho-n) \pi / 2]-z t .
$$

Here, $n=\operatorname{Int}[\rho]$ is the integer value of the density. The EoS is constructed to have the correct chemical potential gap $\Delta \mu_{n}$ built in as well as the correct critical coupling $U_{n}$. Therefore the EoS and resulting phase diagrams are expected to be more accurate than, e.g., calculations within the mean field approximation. The compressibility $d \rho / d \mu$ vanishes in the MI but diverges for densities approaching the MI as in [27]. For $U \gg t$ it has the same EoS as that for fermions, namely that of a non-interacting gas of a single fermion species. The strong on-site repulsion acts as Pauli-blocking similar to the case of strongly correlated nuclear liquid at high densities. The EoS can give the 1D Bose phase diagram at least qualitatively and generalizes the one shown in [29].

The critical radii for the MI transitions can now be calculated in the same way as for fermions by inserting Eq. (16) in Eq. (1). In the strong coupling limit $(t \rightarrow 0)$ the Boson EoS of Eq. (16) becomes exact and we find

$$
R_{n}^{+}=\sqrt{n U / V_{2}}
$$

and

$$
R_{n}^{-}=\sqrt{\left[n U-\Delta \mu_{n}\right] / V_{2}} .
$$

The corresponding critical number of particles $N_{n}^{ \pm}=$ $(2 / d) \int_{0}^{R_{n}^{ \pm}} \rho(r) d r$ are shown in Fig. 2. When $U \gg t$ and $n \gg 1$

$$
N_{n}^{+}=N_{n+1}^{-}=\frac{4 n}{3 d} \sqrt{\frac{n U}{V_{2}}} .
$$

Note, that $R_{1}^{-}=R_{c}$ and $N_{1}^{-}=N_{c}$ for $U \gg t$.

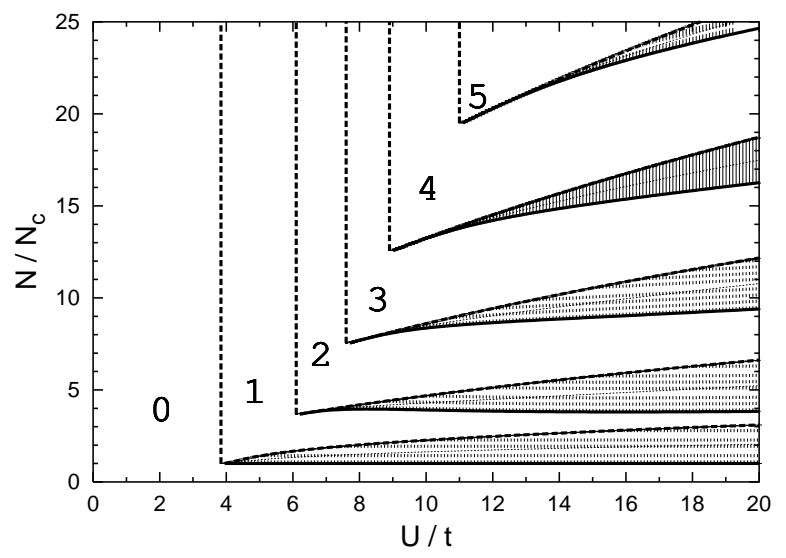

FIG. 2. Phase diagram for 1D bosons with EoS of Eq. (16). The parameter regions numbered $n=0,1,2,3, .$. form $n$ MI phases of density $\rho=(0), 1,2, . ., n$ separated by $n+1$ metal regions between core and surface. In the hatched areas the density has a MI core $\rho(0)=1,2,3, .$. , from below and up.

\section{Higher dimensions}

In higher dimensions $D=2,3$ the radii and critical fillings may be estimated from the scale of the energy and chemical potential. At densities up to unit filling the chemical potential varies monotonously from $-2 z t$ up to 0 or $+2 z t$ in the weak and strong coupling limits respectively. The chemical potential thus varies on a scale $\sim t$ and therefore the size of the system remains 
of order $R_{1}^{-} \sim \sqrt{2 z t / V_{2}}$. Above unit filling the chemical potential gaps are of order $\sim U$ when $U \gg t$ and therefore the scale for the critical radii are unchanged

$$
R_{n+1}^{-}=R_{n}^{+}=\sqrt{n} R_{u} \quad, U \gg t,
$$

where $R_{u}=\sqrt{U / V_{2}}$ is the critical radius for strong couplings (see Eqs. (17-18)). The corresponding critical number of particles is

$$
N_{n+1}^{-}=N_{n}^{+}=\frac{1}{d^{D}} \int \rho(r) d^{D} \mathbf{r}=N_{u} \sum_{m=0}^{n} m^{D / 2},
$$

where $N_{u}=\kappa_{D}\left(R_{u} / d\right)^{D}$, with geometrical factor $\kappa_{D}=$ $2, \pi, 4 \pi / 3$ in $D=1,2,3$ dimensions respectively.

The phase diagram may have additional phases as in the case of the 2D Hubbard model, which is believed to describe high temperature superconductivity. With extended interactions and hopping more MI phases at fractional filling [12] will appear which may separate into supersolid phases [10]. Mixing bosons and fermions leads to a number of new phases [13]. Spin imbalance inhibits pairing (as isospin asymmetry in atomic nuclei) but may also lead to novel pairing phases $[32,33]$.

\section{High temperature superconductivity}

If high $\mathrm{Tc}$ is described by the 2D Hubbard model $[27,8]$, then we can expect that the MI is replaced by an AFM phase around $\rho=1$ surrounded by two dSC phases at densities lower (underdoped) and higher (overdoped). According to [30] phases with AFM and dSC order are mixed when $U \lesssim 8 t$ but coexist when $U \gtrsim 8 t$ with a first order transition between two densities. A critical point is predicted where a mixed $\mathrm{AFM}+\mathrm{dSC}$ phase terminate and is replaced by a first order phase transition. Here the AFM and dSC phases are separated and coexist with a density discontinuity. In [31], however, the pure dSC undergo a first order transition to a mixed AFM + dSC phase. The densities at which these transitions take place are around doping $x=1-\rho \simeq \pm 0.1$. If nearest-neighbor interactions or next-nearest-neighbor hopping are included, the particle-hole symmetry is broken and the phase diagram becomes asymmetric around half filling $(\rho=1)$.

The corresponding phases in confined optical lattices will therefore be much more complex than the 1D shown in Fig. 1. For example, the LL phases will be replaced by metal, dSC and AFM phases and mixed phases. According to [30] there is a also a mixed $\mathrm{dSC}+\mathrm{AFM}$ phase between the pure dSC and AFM phases when $U \lesssim 8 t$ whereas above the dSC and AFM is separated by a first order transition line. The associated density discontinuity is in stark contrast to the constant density MI phase.

Whereas the details of the phases around the MI and AFM shells are not well known, the surrounding shells of dSC phases are again surrounded by two metal phases extending to the surface and the BI core respectively. It would most interesting to study this phase diagram for the 2D Hubbard model on the lattice and to find these phases and possible other phases such as spin glass, strange metal, striped, checker board, etc., and see how they vary with coupling $U / t$, density and temperature.

\section{OBSERVABLES}

The phases described above can be observed by time-of-flight expansion, interference, noise correlations, molecule formation, [1-4], collective modes [34,36,37], rotation [32], etc. We discuss density distributions and radii, collective modes and rotation below.

\section{A. MI shell radii}

The density distribution has recently been measured $[5,6]$ for 3 -D bosons and the MI plateaus observed directly up to $n=5$. The radii of the MI shells were measured for different atomic numbers for a large coupling [5].

In the strong coupling regime the radii of the MI shells $n=1,2, \ldots$ are (see Eq. (20) and Ref. [35])

$$
R_{n}^{2}=R_{1}^{2}-(n-1) R_{u}^{2} .
$$

Note that the radii $R_{n}$ are continous functions of filling whereas the critical radii $R_{n}^{ \pm}$are related to a critical filling $N_{n}^{ \pm}$.

The corresponding particle number in $\mathrm{D}$ dimensions is

$$
N=N_{u} \sum_{n=0}\left(\frac{R_{1}^{2}}{R_{u}^{2}}-(n-1)\right)^{D / 2} .
$$

For bosons the upper limit in the sum is given by $n-1 \leq$ $\left(R_{1} / R_{u}\right)^{2}$ such that $R_{n} \geq 0$. For fermions also $n \leq 2$.

The radii and particle number can now be related universally, i.e. independent of $U, t, V_{2}$ and $d$ separately as long as we are in the strong coupling regime, when scaled by $R_{u}$ and $N_{u}$ respectively. The curves are shown in Fig. 3 for the 3 -D case at zero temperature. In the recent experiment of [5] a 3D optical lattice is filled with ${ }^{87} R b$ atoms in the strong coupling regime such that $R_{u} / d=\sqrt{U / V_{2} d^{2}} \simeq 26$. The measured radii vs. filling is also shown for comparison in Fig. 3 and are in good agreement with theory. 


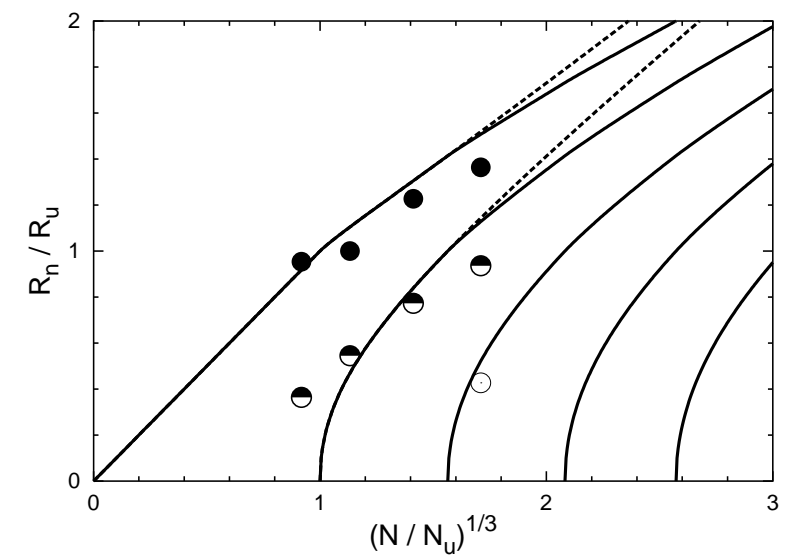

FIG. 3. Radii $R_{n}$ for MI shells vs. number of particles for a $3 \mathrm{D}$ optical lattice. Full curves from left to right show the $n=1,2, . .5$ shell radii for bosons. The dashed curves show the MI $n=1$ and BI $n=2$ for fermions. The solid, half filled and hollow data points are the first three bose MI radii measured in Ref. [5].

\section{B. Collective modes}

The collective modes has proven very useful observable in traps [34,36,37] and we will therefore calculate the modes for optical lattices.

The equations of motion are in hydrodynamics and for a superfluid given by the equation of continuity and the Euler equation. Linearizing around equilibrium these equations lead to $[23,37]$

$$
-m \omega^{2} \mathbf{v}=\frac{1}{\rho} \nabla\left[\rho^{2} \frac{d \mu}{d \rho} \nabla \cdot \mathbf{v}\right]+\nabla(\mathbf{f} \cdot \mathbf{v})-\mathbf{f}(\nabla \cdot \mathbf{v}),
$$

for the flow velocity $\mathbf{v}$. Here, the force from the harmonic trap potential is $\mathbf{f}=-\nabla\left(V_{2} r^{2}\right)=-m \omega_{0}^{2} \mathbf{r}$, where we have introduced the trap frequency $\omega_{0}$ through $V_{2}=m \omega_{0}^{2} / 2$.

The collective modes can be calculated analytically from Eq. (24) when the EoS is a simple polytrope: $\mu(\rho)-\mu(0) \propto \rho^{\gamma}$. At low densities the EoS optical lattices is on such a form for fermions with $\gamma=2 / D$. In 1D $\gamma=2$ for both fermions and bosons according to Eq. (3) and (16). At higher densities the effective polytrope $\bar{\gamma} \equiv[\rho /(\mu-\mu(0))] d \mu / d \rho$ taken at central density $\rho(0)=\rho_{c}$ gave rather accurate collective modes in atomic traps $[36,37]$. For 1D fermions in an optical lattice we find from Eq. (3)

$$
\bar{\gamma}=\frac{\pi \rho_{c}}{\beta} \cot \left(\pi \rho_{c} / 2 \beta\right),
$$

for $\rho_{c}<1$. In the weakly interacting and the attractive cases $U \leq 0$ with EoS given by Eq. (13), we also obtain Eq. (25) with $\beta=2$.

The collective modes of fermions and bosons are the same in the strong coupling limit since bosons with EoS of Eq. (16) also has the effective polytrope of Eq. (25) with $\beta=1$. In contrast a dilute bose $\operatorname{EoS}\left(U \ll U_{1}\right)$ has $\bar{\gamma}=1$ (see Fig. 3). We can expect a continuous change of $\bar{\gamma}=1$ between these two limits. When $\rho_{c} \rightarrow 1$ and $U \gg t$ both bosons and fermions have $\bar{\gamma} \rightarrow 0$ whereas at $\rho_{c}=1$ a MI forms in the center making the system incompressible (corresponding to $\bar{\gamma} \rightarrow \infty$ ).

As in $3 \mathrm{D}$ [37] the collective modes can be calculated in $1 \mathrm{D}$ generally for any polytropic EoS. Eq. (24) reduces to

$$
\frac{1}{2}\left(1-x^{2}\right) \frac{d^{2} v}{d x^{2}}-(\gamma+1) x \frac{d v}{d x}+\left(\frac{\omega^{2}}{\omega_{0}^{2}}-1\right) v=0,
$$

where $x=r / R$. The eigenfunctions are $v \propto F(-n, n+$ $2 \gamma-1, \gamma+1,2 x-1)$, where $F$ is the hypergeometrical function. The corresponding $1 \mathrm{D}$ eigenvalues for multipoles with $n=0,1,2, \ldots$, nodes are

$$
\frac{\omega^{2}}{\omega_{0}^{2}}=1+n[\gamma+1+(n-1) / 2] .
$$

The dipole mode $(n=0)$ has constant flow velocity in all dimensions and by insertion in Eq. (24) has frequency $\omega / \omega_{0}=1$. It is independent of dimension, $\operatorname{EoS}(\gamma)$ and central density because the dipole mode corresponds to the whole cloud sloshing as a rigid body in the trap and thus only depends on the trap geometry in one direction (as long as $\rho_{c}<1$ ). The breathing or monopole mode frequency has flow velocity $\mathbf{v} \propto \mathbf{r}$ and by insertion in Eq. (24) the monopole frequency is found to be $\omega^{2} / \omega_{0}^{2}=$ $2+\gamma D$, generally in $D$-dimensions.

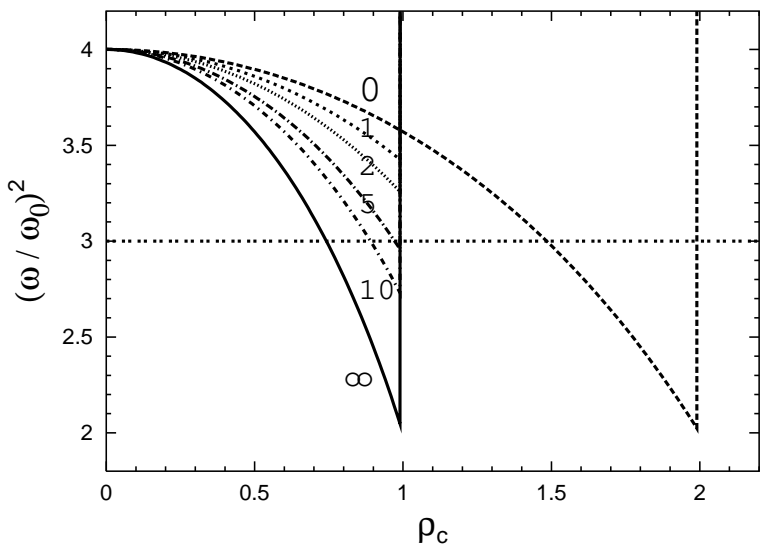

FIG. 4. 1D breathing mode frequency $\omega^{2} / \omega_{0}^{2}=2+\bar{\gamma}$ vs. central density. The modes for fermions are labelled with $U / t=0,1,2,5,10, \infty$. Full curve gives the strong coupling limit $U \gg U_{1}$ for both fermions and bosons. The weak coupling limit $U \simeq 0$ is shown by dashed curve for fermions and dotted curve for bosons where $\omega^{2} / \omega_{0}^{2}=3$.

In Fig. 3 we show the $1 \mathrm{D}$ breathing mode frequency $(n=1) \omega^{2} / \omega_{0}^{2}=2+\bar{\gamma}$ with the effective polytrope $\bar{\gamma}$ given by Eq. (25). It decreases with increasing density until the MI appears in the core which is incompressible. Likewise we can expect that the collective modes will reveal the AFM state for the 2D Hubbard model. The dSC 
may only expected to affect the EoS and collective modes weakly. Rotational vortices [32] or RF spectroscopy [38] may therefore be better signals for the dSC phases.

\section{Rotation}

By rotating the atoms in the traps the BEC and BCS superfluids are revealed by vortex formation [32]. Similarly, for confined 2D optical lattices the layers of BEC or dSC superfluids with bosons and fermions respectively may be identified by vortices when rotated. The MI phase is solid and the atoms in it cannot be rotated unless a supersolid phase exists or the lattice itself is rotated.

The possible density discontinuity [30,31] leads to a non-analytic moment of inertia. Due to centrifugal forces the critical radii and fillings now also depends on rotation frequency and therefore the density discontinuity can made to occur right in the center by varying either the particle number or the angular frequency. Such core phase transitions have been considered for neutron stars resulting in a characteristic behavior of neutron star spin down frequencies with time [39].

Solids in confined optical lattices will prefer ellipsoidal deformation at fast rotation and varying the angular velocity may therefore create glitches (MI quakes) as observed for neutron stars.

\section{SUMMARY}

In summary, optical lattices provide a clean system where 1, 2 and 3D Hubbard and Bose-Hubbard models can be realized. The phase transitions between MI, LL, LE, superfluids and metals were calculated in $1 \mathrm{D}$ and in $2+3 \mathrm{D}$ for the strong coupling regime, where comparison was made to recent data on radii and fillings for bose atoms in optical lattices. Collective modes were calculated and found to be sensitive to the underlying EoS and phases. 2D optical lattices with fermions will therefore give direct insight in the high temperature superconductivity and the $\mathrm{dSC}+\mathrm{AFM}$ phases vs. interaction strength, doping and temperature.

[1] M. Greiner et al., Nature 419, 51 (2002).

[2] T. Stöferle et al., Phys. Rev. Lett. 92, 130403 (2004); ibid. 96, 030401 (2006). H. Moritz, T. Stöferle, K. Günter, M. Köhl, T. Esslinger, ibid. 94, 210401 (2005).

[3] F. Gerbier et al., Phys. Rev. A 72, 053606 (2005) and Phys. Rev. Lett. 96, 090401 (2006).

[4] G. Thalhammer et al., Phys. Rev. Lett. 96, 050402 (2006).

[5] S. Fölling, A. Widera, T. Müller, F. Gerbier, I. Bloch, cond-mat/0606592.

[6] G.K. Campbell, J. Mu, M. Boyd, P. Medley, A.E. Leanhardt, L. Marcassa, D.E. Pritchard, W. Ketterle, condmat/0606642.
[7] D.J. Scalapino, Physics Reports 250, 329 (1995). P.W. Anderson, Science 235, 1196 (1987).

[8] W. Hofstetter et al., Phys. Rev. Lett. 89, 220407 (2002)

[9] L.-M. Duan, Phys. Rev. Lett. 95, 243202 (2005). A. Isacsson and S. M. Girvin, cond-mat/0506622

[10] V.W. Scarola and S. Das Sarma, Phys. Rev. Lett. 95, 033003 (2005)

[11] A.S. Sørensen, E. Demler, M.D. Lukin, condmat/0405079.

[12] H. Heiselberg, Phys. Rev. A 73, 013628 (2006).

[13] D.-W. Wang, M.D. Lukin, E. Demler, Phys. Rev. A 72, 051604(R) (2005)

[14] J. Hubbard, Phys. Rev. B 17, 494 (1978).

[15] M.P.A. Fisher, P.B. Weichman, G. Grinstein, D.S. Fisher, Phys. Rev. B 40, 546 (1989).

[16] D. Jaksch et al., Phys. Rev. Lett. 81, 3108 (1998).

[17] W. Zwerger, J. Opt. B 5, 9 (2003).

[18] B. Paredes, P. Fedichev, J.I. Cirac, P. Zoller, Phys. Rev. Lett. 87, 010402 (2001).

[19] V.L. Campo \& K. Capelle, Phys. Rev. A 72, 061602(R) (2005); N.A. Lima, M.F. Silva, L.N. Oliveira, K.Capelle, Phys. Rev. Lett. 90, 146402 (2003).

[20] X.-J. Liu, P.D. Drummond, H. Hu, Phys. Rev. Lett. 94, 136406 (2005).

[21] G. Xianlong et al., cond-mat/0506570; Phys. Rev. B 73, 165120 (2006); Phys. Rev. B 73, 161103(R) (2006).

[22] M. Rigol, A. Muramatsu, Phys. Rev. A 69, 053612 (2004) and Opt. Commun. 243, 33 (2004). M. Rigol et al., Phys. Rev. Lett. 91, 130403 (2003).

[23] C.J. Pethick and H. Smith, Bose-Einstein Condensation in Dilute Gases, Cambridge Univ. Press, 2002.

[24] E.H. Lieb and F.Y. Wu, Phys. Rev. Lett. 20, 1445 (1968).

[25] J.M. Luttinger, J. Math. Phys. N.Y. 4, 1154 (1963).

[26] A. Luther and V.J. Emery, Phys. Rev. Lett. 33, 589 (1974).

[27] G.G. Batrouni, R.T. Scalettar and G.T. Zimanyi, Phys. Rev. Lett. 65, 1765 (1990). P. Niyaz, R.T. Scalettar, C.Y. Fong, and G.G. Batrouni, Phys. Rev. B 44, R7143 (1991)

[28] T.D. Kühner and H. Monien, Phys. Rev. B 58, R14741 (1998). N. Elstner and H. Monien, cond-mat/9905367

[29] C. Kollath, U. Schollwöck, J. von Delft, and W. Zwerger, Phys. Rev. A 69, 031601(R) (2004)

[30] M. Capone and G. Kotliar, cond-mat/0603227.

[31] M. Aichhorn, E. Arrigoni, M. Potthoff, W. Hanke, condmat/0511460.

[32] M.W. Zwierlein et al., Science 311, 492 (2006); Nature 435, 1047 (2005).

[33] G.B. Partridge et al., Science, 23 December 2005 (10.1126/science.1122876) (cond-mat/0511752).

[34] J. Kinast et al., Phys. Rev. Lett. 92, 150402 (2004). M. Bartenstein et al., Phys. Rev. Lett. 92, 203201 (2004)

[35] B. DeMarco, C. Lannert, S. Vishveshwarea, T.-C. Wei, Phys. Rev. A 71, 063601 (2005).

[36] H. Hu, A. Minguzzi, X-J Liu, M.P. Tosi, Phys. Rev. Lett. 93, 190403 (2004).

[37] H. Heiselberg, Phys. Rev. Lett. 93, 040402 (2004).

[38] C. Chin et al., Science 3051128 (2004).

[39] H. Heiselberg, M. Hjorth-Jensen, Phys. Rev. Lett. 80 (1998) 5485. 